\title{
Luminescent Schiff-Base Lanthanide Single-Molecule Magnets: The Association Between Optical and Magnetic Properties
}

\author{
Jérôme Long * \\ Institut Charles Gerhardt, Equipe Ingénierie Moléculaire et Nano-Objets, Université de Montpellier, ENSCM, CNRS, \\ Montpellier, France
}

OPEN ACCESS

Edited by:

Luís António Dias Carlos,

University of Aveiro, Portugal

Reviewed by:

Jose Ramon Galan-Mascaros, Institut Català d'Investigació Química,

Spain

Mahmut Özacar

Sakarya University, Turkey

*Correspondence:

Jérôme Long

jerome.long@umontpellier.fr

Specialty section:

This article was submitted to

Inorganic Chemistry,

a section of the journal

Frontiers in Chemistry

Received: 05 December 2018

Accepted: 23 January 2019

Published: 06 February 2019

Citation:

Long J (2019) Luminescent

Schiff-Base Lanthanide

Single-Molecule Magnets: The

Association Between Optical and

Magnetic Properties.

Front. Chem. 7:63

doi: 10.3389/fchem.2019.00063
Luminescent Single-Molecule Magnets (SMM) belong to a new class of multifunctional molecule-materials that associate luminescence and slow relaxation of their magnetization within a single crystalline phase. We present in this mini-review the major advances that have been achieved in this new field over the last few years. More particularly, we will focus on the use of Schiff-base complexes in order to correlate magnetism and luminescence, as well as discussing the future outlooks of the field.

Keywords: lanthanides, single-molecule magnet, multifunctional molecular materials, luminescence, anisotropy, crystal-field splitting

\section{INTRODUCTION}

Nowadays, developing optimized molecule-based materials for future applications such as biomedicine (Horcajada et al., 2012; Long et al., 2016a), gas separation (Dechambenoit and Long, 2011), catalysis (Li et al., 2016), and quantum computing (Bogani and Wernsdorfer, 2008) frequently requires the association of different properties within a single-crystalline structure. Molecule-based materials benefit from specific assets related to their molecular nature, with respect to usual solid-state materials such as an unlimited structural diversity, weak density, optical transparency and the possibility to finely adjust and control their properties. Remarkably, coordination chemists have been at the front lines of science since the turn of the century, taking advantage of the respective intrinsic properties of metal ions and organic/inorganic ligands, to design original architectures with targeted functionalities. From a fundamental point of view, these unique molecular materials may display these properties independently, but the design of systems in which these functionalities strongly interact, constitutes the central objective for one property to control another.

Since the pioneering work of (Ishikawa et al., 2003), lanthanide-based Single-Molecule Magnets (SMM) have been investigated thoroughly because of their tremendous technological potential in high-density storage and quantum computing (Leuenberger and Loss, 2001; Woodruff et al., 2013; Liddle and van Slageren, 2015; Tang and Zhang, 2015; Ungur and Chibotaru, 2016). In such coordination complexes, an anisotropic barrier, $\Delta$, originating from the interplay between the magnetic anisotropy and crystal-field splitting, opposes the reversal of the magnetization and leads to superparamagnetic-like behavior, comparable to that observed in magnetic nanoparticles. This feature may eventually give rise to a magnetic bistability that is strictly intrinsic to the molecular entity. Obviously, utilizing lanthanide ions constitutes a straightforward approach to implement simultaneous magnetic and luminescent properties because of their strong magnetic anisotropy and exceptional luminescence properties, dominated by $f-f$ electronic 
transitions, which results in long-lived emission, narrow bandwidth, important Stokes shifts and high quantum yields (Bunzli and Piguet, 2005). While the collection of lanthanide SMM is growing exponentially, only a small percentage of those systems exhibit lanthanide luminescence and can therefore be viewed as multifunctional. In this mini-review, we discuss the use of Schiff-base ligands and associated complexes for the design of luminescent SMM, as well as providing future outlooks and directions in the field.

\section{CRITERIA TO DESIGN LANTHANIDE LUMINESCENT SMM}

Slow relaxation of the magnetization and lanthanide luminescence, arises in both cases from the subtle association between a defined lanthanide ion and appropriate ligand(s). Therefore, the SMM behavior depends on the nature of the lanthanide ion, such as its angular momentum value, $J$, its Kramers/non-Kramers character as well as the angular dependence of the $4 f$ electronic density which can be oblate (flattened spheroid) or prolate (elongated spheroid) (Rinehart and Long, 2011; Ungur and Chibotaru, 2016). On the other hand, and considering simple electrostatic considerations, the crystal-field generated by the surrounding ligands could result in the formation of $m_{\mathrm{J}}$ states largely separated in energy. Since it is mostly the single-ion anisotropy that dominates the slow relaxation, $\Delta$ is therefore directly related to crystalfield splitting, through relaxation involving the first or higher excited states. Other mechanisms involved in spin-phonon coupling (Raman and direct processes) or Quantum tunneling of Magnetization (QTM) complicate this scenario however, by creating underbarrier relaxation paths. In this sense, significant advances have been achieved in recent years, with either coordination (Chen et al., 2016; Liu et al., 2016; Meng et al., 2018) or organometallic complexes (Chen et al., 2016; Ding et al., 2016; Gregson et al., 2016; Gupta et al., 2016; Goodwin et al., 2017; Guo et al., 2017) showing for instance magnetic hysteresis higher than liquid nitrogen boiling's temperature (Guo et al., 2018). Remarkably, while the QTM affects the magnetic relaxation at a low temperature, recent studies have highlighted the decisive role of molecular vibrations (spin-phonon coupling) at a higher temperature (Goodwin et al., 2017; Escalera-Moreno et al., 2018).

With regards to lanthanide luminescence, the parity and spin forbidden character of the $f-f$ transitions usually require an indirect excitation through the use of sensitizer ligands that transfers the absorbed energy to the excited state of the lanthanide ion. Consequently, the ligand is of utmost importance since it directly dictates the coordination environment suitable for the slow relaxation of the magnetization, while ensuring an efficient luminescence sensitizing toward a specific lanthanide ion. Among the lanthanide series, $\mathrm{Dy}^{3+}$ ion represents one of the most promising candidates to design luminescent SMM, because of its large $J=15 / 2$ value, its Kramers character leading to a doubly degenerated ground state and its oblate electronic density which could be easily stabilized by usual coordination chemistry ligands. $\mathrm{Dy}^{3+}$ luminescence could be observed both in the visible and Near-Infra Red (NIR) (Long et al., 2018b). To a lesser extent, the NIR emissive $\mathrm{Yb}^{3+}$ has also been widely employed to design luminescent SMM (Pointillart et al., 2015), but optimizing the slow relaxation remains more difficult to realize with respect to $\mathrm{Dy}^{3+}$. $\mathrm{Tb}^{3+}$ and $\mathrm{Er}^{3+}$ could also be employed to design luminescent SMM. Nevertheless, in practice they exhibit some drawbacks associated either to the $\mathrm{Tb}^{3+}$ non-Kramers nature (Ehama et al., 2013; Yamashita et al., 2013) or to the difficulty to observe $\mathrm{Er}^{3+}$-based luminescence (Ren et al., 2014). Historically, the first example of SMM simultaneously exhibiting a slow relaxation of the magnetization and a weak lanthanide luminescence was reported by $\mathrm{Bi}$ et al. in a tetranuclear calixarene dysprosium complex in 2009 (Bi et al., 2009). Following this, various ligand families (beta-diketonates, carboxylates, aromatic amines) have been successfully explored (Long et al., 2018b; Jia et al., 2019). We will however, next focus on another class belonging to Schiff bases and describe examples that go further than the simple observation of both properties.

\section{LUMINESCENT SCHIFF-BASE SMM: MAGNETO-LUMINESCENCE CORRELATION}

Schiff-base ligands are known as simple efficient sensitizers of $\mathrm{Ln}^{3+}$ (Yang et al., 2014; Andruh, 2015) while benefiting from a large tunability including the denticity, rigidity/flexibility, and selective coordination sites (Figure 1). Several $4 f$ or $3 d / 4 f$ luminescent complexes based on a myriad of Schiff-base ligands and incorporating various lanthanide ions such as $\mathrm{Eu}^{3+}$, $\mathrm{Nd}^{3+}, \mathrm{Tb}^{3+}, \mathrm{Yb}^{3+}$, have been reported since the beginning of the century (Wong et al., 2002, 2006; Yang and Jones, 2005; Burrow et al., 2009; Wang et al., 2009). Nevertheless, the occurrence of slow relaxation of magnetization in these complexes was either not achieved, due to the lack of magnetic anisotropy, or was not investigated. Thus, the first example of a bifunctional Schiff-base SMM was reported by our group in 2012 in a $\left[\mathrm{Zn}\left(\mathrm{NO}_{3}\right) \mathrm{L}^{1} \mathrm{Dy}\left(\mathrm{NO}_{3}\right)_{2}\left(\mathrm{H}_{2} \mathrm{O}\right)\right]$ complex $\left[\mathrm{H}_{2} \mathrm{~L}^{1}\right.$ : $\mathrm{N}, \mathrm{N}$ '-bis(3-methoxysalicylidene)-1,2-diaminoethane)] and based on a simple compartmentalized ligand obtained from the condensation of $o$-vanillin and ethylenediamine (Long et al., 2012). The complex could be described as a dinuclear entity in which the connections between $\mathrm{Zn}^{2+}$ and $\mathrm{Dy}^{3+}$ ions are provided by phenolate bridges (Figure 2). Introduction of the diamagnetic $\mathrm{Zn}^{2+}$ ion clearly has two benefits: it increases the negative charge (basicity) of the phenolate moieties and in turn the crystal-field splitting of the lanthanide ion (Upadhyay et al., 2014) while it does not quench the rare-earth emission in the visible spectral window. At room temperature, both a broad emission band, ascribed to the zinc complex and the typical $\mathrm{Dy}^{3+}$ emission lines were observed, indicating a partial energy transfer toward the lanthanide ion. Lowering the temperature $(14 \mathrm{~K})$ results in the exclusive observation of well-resolved $\mathrm{Dy}^{3+}$ emission bands. Such feature is of prime interest since the emission lines involving the electronic transitions also involve the magnetic ground state ${ }^{6} \mathrm{H}_{15 / 2}$ and 
as a consequence directly reflects its crystal-field splitting. Remarkably, such an approach was previously utilized in the 60's, using absorption spectroscopy for ytterbium (Buchanan et al., 1967) or dysprosium garnets (Grünberg et al., 1969) and this methodology was later extended by Cucinotta et al. (2012) to correlate luminescence and magnetic properties in the archetypical SMM Na[Dy(DOTA) $\left.\left(\mathrm{H}_{2} \mathrm{O}\right)\right] \cdot 4 \mathrm{H}_{2} \mathrm{O}$ complex (Cucinotta et al., 2012). The emission spectrum for the complex $\left[\mathrm{Zn}\left(\mathrm{NO}_{3}\right) \mathrm{L}^{1} \mathrm{Dy}\left(\mathrm{NO}_{3}\right)_{2}\left(\mathrm{H}_{2} \mathrm{O}\right)\right]$ shows more than eight expected transitions, resulting from the splitting of ${ }^{6} \mathrm{H}_{15 / 2}$ ground state into eight Kramers doublets $(J+1 / 2)$. This indicates the presence of "hot bands" arising from the first excited state of the emitting level ${ }^{7} \mathrm{~F}_{9 / 2}$ (Figure 2). Deconvolution of the emission bands, using Gaussian functions, allows one to experimentally obtain the crystal-field splitting of the Kramers doublets. Hence, the gap between the ground and first excited doublets is estimated at $48 \mathrm{~cm}^{-1}$, in line with the value of $\Delta=35 \mathrm{~cm}^{-1}$ obtained by alternate currents (ac) magnetic measurements. This suggests that the relaxation occurs via the first excited Kramers doublet corresponding to an Orbach process. Nevertheless, the slight discrepancy between luminescence and magnetism indicates that additional magnetic relaxation mechanisms are involved. This is further corroborated through the study of both the magnetic and luminescence properties of the aforementioned complex, diluted in a diamagnetic yttrium matrix (Long et al., 2016b). Photoluminescence confirms that: (i) the $\mathrm{Dy}^{3+}$ ion remains in a similar environment upon chemical dilution; (ii) as expected, the energy gap between the ground and first excited doublets is identical. The magnetic measurements for the diluted sample reveal an increased anisotropic barrier of $\Delta=45 \mathrm{~cm}^{-1}$ due to the removal of the dipolar interactions, known to enhance the QTM, that decrease the effective barrier. The value of $\Delta$ is in remarkable accordance with that obtained by luminescence, which confirms that such an approach can be used to compare the results from these two experimental techniques and further shed light on the mechanisms that govern the slow relaxation of magnetization.

Such simple methodology has been widely extended, by other groups and including our own, to numerous SMM or coordination networks that exhibit a single-ion based magnetic relaxation in order to obtain a detailed picture of the lanthanide crystal-field and therefore improves comprehension of the relaxation dynamics (Long et al., 2018b; Jia et al., 2019). We would also like to emphasize that such outcomes could also be further confirmed by the decisive input from $a b$ initio calculations, especially with systems that exhibit multiple crystallographically independent sites (Long et al., 2015).

Pure $4 f$ Schiff base complexes also represent another class of promising systems. However, it remains difficult to simultaneously increase the magnetic anisotropy to generate a genuine slow relaxation of magnetization, while retaining the lanthanide luminescence (Shintoyo et al., 2014; Long et al., 2018a). Therefore, one alternative strategy consists of synthesizing heterotrinuclear $\mathrm{Zn}_{2}$ Dy complexes (Oyarzabal et al., 2015; Sun et al., 2016) in which the lanthanide ion is sandwiched between two bis-phenoxide moieties. This results in

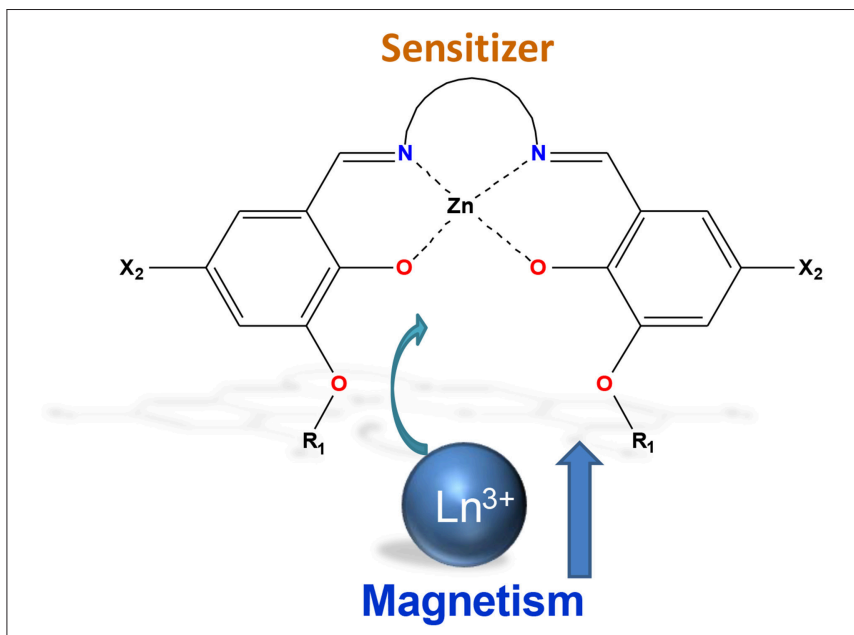

FIGURE 1 | General scheme showing the combination of compartmental Schiff-base complex and $\mathrm{Ln}^{3+}$ ions.

an enhancement of the axial crystal-field. The five-membered ring constituted by four methoxy oxygen and one solvate, or counter-ion, defines a basal plane (hard plane) almost perpendicular to the $\mathrm{Zn}^{2+}-\mathrm{Dy}^{3+}-\mathrm{Zn}^{2+}$ arrangement. Numerous complexes with various counter-anions and co-ligands that exhibit SMM behavior and $\mathrm{Dy}^{3+}$-based luminescence associated with remanent emission from the ligands, have been reported (Costes et al., 2015, 2016). To this point, we have recently described an example of a luminescent trinuclear complex $\left[\left(\mathrm{ZnL}^{1} \mathrm{Cl}\right)_{2} \mathrm{Dy}\left(\mathrm{H}_{2} \mathrm{O}\right)\right]_{4}\left[\mathrm{ZnCl}_{4}\right]_{2} \cdot \mathrm{H}_{2} \mathrm{O}$ that shows a zero-field slow relaxation of magnetization that could be observed for up to $30 \mathrm{~K}$ (Boulkedid et al., 2018). The compound exhibits the typical Dy ${ }^{3+}$ emission, but the presence of four different crystallographically independent dysprosium sites precludes the extraction of the energy difference between the ground and first excited doublets. Luminescence reveals however a large total crystal-field splitting of about $1,500 \mathrm{~cm}^{-1}$, indicating that such systems may have great potential if the relaxation occurs through higher excited Kramers doublets.

Schiff-base ligands could also be employed to introduce further functionalities. For instance, implementing chirality, opens the field to new properties that result from the crystallization in non-centrosymmetric structures. One can cite for instance Natural Circular Dichroism (NCD), SecondHarmonic Generation (SHG), Circular Polarized Luminescence $(\mathrm{CPL})$ as well as magneto-optical cross-effects that result from the interplay with magnetism, such as magneto-chiral dichroism and Magnetized Second-Harmonic Generation (MSHG) (Train et al., 2011). In addition, the crystallization in appropriate non-centrosymmetric space groups paves the way toward advanced electrical properties such as piezo/pyroelectricity and ferroelectricity. With this in mind, we reported chiral $\left[\mathrm{ZnL}{ }^{2} \mathrm{Dy}(\mathrm{OAc})\left(\mathrm{NO}_{3}\right)_{2}\right]$ complexes based on the enantiopure Schiff base ligands $R, R$ or $S, S-\mathrm{HL}_{2}=$ phenol,2,2' [2,2-diphenyl1,2-ethanediyl]bis[(E)-nitrilomethylidyne]-bis(6-methoxy)

(Long et al., 2015). Each enantiomer crystallizes in the polar 


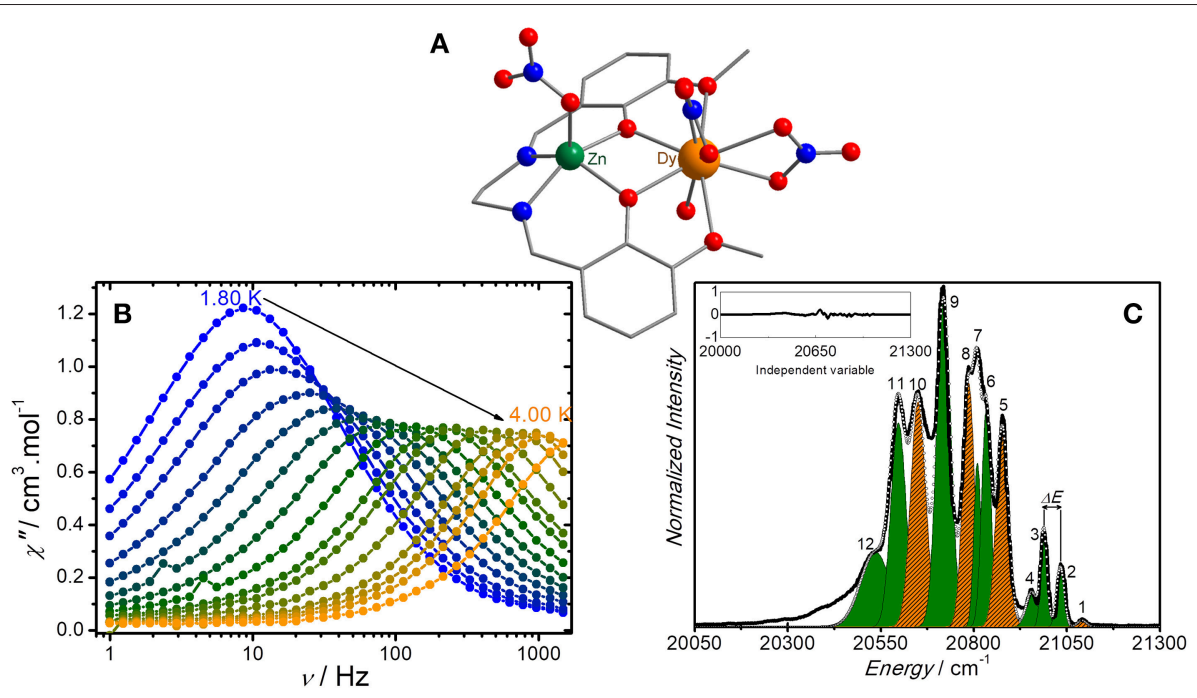

FIGURE 2 | (A) Crystal structure of the dinuclear $\left[\mathrm{Zn}\left(\mathrm{NO}_{3}\right) L^{1} \mathrm{Dy}\left(\mathrm{NO}_{3}\right)_{2}\left(\mathrm{H}_{2} \mathrm{O}\right)\right]$ complex. (B) Frequency dependence of the out-of-phase susceptibility ( $\chi$ ") obtained under a 900 dc field. (C) Magnification of the ${ }^{4} \mathrm{~F}_{9 / 2} \rightarrow{ }^{6} \mathrm{H}_{15 / 2}$ emission transitions in the 20,050-21,300 $\mathrm{cm}^{-1}$ region (at $14 \mathrm{~K}$ ) Multi-Gaussian function fit components arising from the first ${ }^{4} \mathrm{~F}_{9 / 2}$ Stark sublevel to the ${ }^{6} \mathrm{H}_{15 / 2}$ multiplet in the energy interval. The fits regular residual plots $\left(R^{2}>0.98\right)$ are shown in inset (Long et al., 2012, 2016b).

$P 2_{1}$ space group, with two crystallographically inequivalent homochiral $\mathrm{Zn}^{2+} / \mathrm{Dy}^{3+}$ complexes, in the asymmetric unit. Apart from the typical dysprosium luminescence and slow relaxation of the magnetization that have been correlated and compared with results obtained from $a b$ initio calculations, the compounds exhibit a ferroelectric behavior up to the decomposition of the material at $300^{\circ} \mathrm{C}$, making it the highest temperature at which a switchable polarization has been observed for a molecular ferroelectric. Such robust chiral molecular compounds may represent alternative candidates for high-temperature ferroelectrics (Hang et al., 2011).

\section{CONCLUSIONS AND FUTURE OUTLOOK}

Schiff-bases represent an interesting class of ligands from which to design luminescent lanthanide SMM. Their infinite diversity and flexibility make them ideal candidates to face the challenges in the field and to obtain air-stable luminescent SMM with high energy barriers. In a more general context, the in-depth understanding of magnetic relaxation in lanthanidebased SMM remains a challenge as this involves concepts and models in physics from the 60's, related for instance to spinphonon coupling (Escalera-Moreno et al., 2018), which should be modernized. In this regard, photoluminescence could definitely shed light on lanthanide crystal-field splitting, to determine if the relaxation proceeds via the 1st or higher excited states, or involves underbarrier Raman, direct or QTM processes. Moreover, studying the vibronic coupling in high temperature SMM may be experimentally achieved by photoluminescence (emission lifetimes, $f-f$ relative intensities...).
While the correlation between magnetism and photoluminescence should be viewed as the inception, studying the interplay between the two properties constitutes a major milestone. As both properties are intimately correlated to the electronic structure of the lanthanide ion, the coupling is expected to be strong and such a cross-effect has previously been evidenced more than 50 years ago, in paramagnetic ytterbium garnet (Buchanan et al., 1967; Grünberg et al., 1969). Thus, applying a magnetic field induces a strong modification of the emission spectrum and has been explained by the well-known Zeeman effect, that lifts the degeneracy of the Kramers doublets. Such approach was first demonstrated in lanthanide SMM, in 2016 (Bi et al., 2016) in which values of the gyromagnetic factor (that are usually difficult to experimentally obtain) were extracted, confirming the gap between the first and excited Kramers doublets. One point that needs to be addressed concerns the comprehension of the field dependence of the emission intensity as observed in others SMM (Chen et al., 2017). Such breakthroughs confirm the possibility to control both the emission intensity and wavelength by a magnetic field, which could be relevant for applications such as magnetic field sensors. However, such inductive effects may simply occur in any luminescent $4 f$ paramagnetic compound, therefore, future studies should examine the existence of an interplay between the SMM property (magnetic bistability) and the luminescence. This requires circumventing synthetic issues to design systems, simultaneously exhibiting a significant magnetic remanence and coercivity at a pertinent temperature with stability over a long-time scale. This last point is of critical importance since $4 f$ SMM usually shows quick relaxation of magnetization, due to the QTM. Major advances have recently been achieved in organometallic 
SMM, showing magnetic bistability of up to $60-80 \mathrm{~K}$ (Goodwin et al., 2017; Guo et al., 2017, 2018), confirming that the design of high performing luminescent SMM with possible air-stability is within reach if suitable sensitizer ligands that are able to maximize the magnetic anisotropy, are rationally conceived.

On the other hand, introduction of chirality and other properties, resulting from the non-centrosymmetric character of the crystal structures, may be easily achieved using Schiff base ligands. Simple multifunctional molecular ferroelectrics therefore represent ideal candidates to study the coupling between the constitutive functionalities such as the magnetoelectrical coupling. Designing a strong coupling between an electric and magnetic property clearly constitutes an important challenge in the field of solid-state chemistry (Fiebig et al., 2016). This indicates that molecular systems may control polarization, by applying a magnetic field and vice versa with prospective applications in non-volatile memories and low-consumption devices (Eerenstein et al., 2006; Cheong and Mostovoy, 2007; Mandal et al., 2015).

More generally and in order to fulfill these ambitious objectives, molecular materials also need to be integrated or shaped into more complex architectures (surfaces, films, or composites) for use in practical applications. Such strategies necessitate investigating that the considered molecular objects and their associated functionalities remain preserved. Among the characterization techniques used to investigate the latter,

\section{REFERENCES}

Andruh, M. (2015). The exceptionally rich coordination chemistry generated by Schiff-base ligands derived from o-vanillin. Dalton Trans. 44, 16633-16653. doi: 10.1039/C5DT02661J

Bi, Y., Chen, C., Zhao, Y.-F., Zhang, Y.-Q., Jiang, S.-D., Wang, B.-W., et al. (2016). Thermostability and photoluminescence of Dy(iii) single-molecule magnets under a magnetic field. Chem. Sci. 7, 5020-5031. doi: 10.1039/C6SC01157H

Bi, Y., Wang, X.-T., Liao, W., Wang, X., Deng, R., Zhang, H., et al. (2009). Thiacalix[4]arene-Supported planar Ln 4 (Ln = TbIII, DyIII) clusters: toward luminescent and magnetic bifunctional materials. Inorg. Chem. 48, 11743-11747. doi: 10.1021/ic9017807

Bogani, L., and Wernsdorfer, W. (2008). Molecular spintronics using singlemolecule magnets. Nat. Mater. 7, 179-186. doi: 10.1038/nmat2133

Boulkedid, A.-L., Long, J., Beghidja, C., Guari, Y., Beghidja, A., and Larionova, J. (2018). A luminescent Schiff-base heterotrinuclear Zn2Dy singlemolecule magnet with an axial crystal field. Dalton Trans. 47, 1402-1406. doi: 10.1039/C7DT03842A

Buchanan, R. A., Wickersheim, K. A., Pearson, J. J., and Herrmann, G. F. (1967). Energy levels of $\mathrm{Yb}^{3+}$ in gallium and aluminum garnets. I. spectra. Phys. Rev. $159,245-251$.

Bunzli, J.-C. G., and Piguet, C. (2005). Taking advantage of luminescent lanthanide ions. Chem. Soc. Rev. 34, 1048-1077. doi: 10.1039/B40 $6082 \mathrm{M}$

Burrow, C. E., Burchell, T. J., Lin, P.-H., Habib, F., Wernsdorfer, W., Clérac, R., et al. (2009). Salen-Based [Zn2Ln3] complexes with fluorescence and single-molecule-magnet properties. Inorg. Chem. 48, 8051-8053. doi: $10.1021 /$ ic 9007944

Chen, Y.-C., Liu, J.-L., Ungur, L., Liu, J., Li, Q.-W., Wang, L.-F., et al. (2016). Symmetry-supported magnetic blocking at $20 \mathrm{~K}$ in pentagonal bipyramidal Dy(III) single-ion magnets. J. Am. Chem. Soc. 138, 2829-2837. doi: $10.1021 /$ jacs.5b13584 photo-luminescence could easily be used for this purpose. Close collaboration between chemists and photo physicists is therefore clearly necessary, in order to achieve these objectives.

\section{AUTHOR CONTRIBUTIONS}

The author confirms being the sole contributor of this work and has approved it for publication.

\section{FUNDING}

I thank the University of Montpellier, CNRS, LABEX CheMISyst ANR-10-LABX-05-01 and the Portugal-France bilateral action Program PESSOA (Hubert Curien) Multifunctional magnetoluminescent molecular architectures (33676SF) and Magnetooptical Multifunctional Nanoparticles (40773PE) for financial support.

\section{ACKNOWLEDGMENTS}

I would like to thank in particular Dr. Y. Guari and Pr. J. Larionova for their expert advice and fruitful scientific discussions. I also deeply acknowledge my colleagues Pr. R. A. S. Ferreira and Pr. Luis D. Carlos for the great collaboration. I also express my deepest acknowledgments to all my co-workers, colleagues and students.

Chen, Y. C., Liu, J. L., Lan, Y., Zhong, Z. Q., Mansikkamaki, A., Ungur, L., et al. (2017). Dynamic magnetic and optical insight into a high performance pentagonal bipyramidal dyIII single-ion magnet. Chem. Eur. J. 23, 5708-5715. doi: 10.1002/chem.201606029

Cheong, S.-W., and Mostovoy, M. (2007). Multiferroics: a magnetic twist for ferroelectricity. Nat. Mater. 6, 13-20. doi: 10.1038/nmat1804

Costes, J. P., Titos-Padilla, S., Oyarzabal, I., Gupta, T., Duhayon, C., Rajaraman, G., et al. (2015). Analysis of the role of peripheral ligands coordinated to znii in enhancing the energy barrier in luminescent linear trinuclear Zn-Dy-Zn single-molecule magnets. Chem. Eur. J. 21, 15785-15796. doi: 10.1002/chem.201501500

Costes, J. P., Titos-Padilla, S., Oyarzabal, I., Gupta, T., Duhayon, C., Rajaraman, G., et al. (2016). Effect of ligand substitution around the DyIII on the SMM properties of dual-luminescent $\mathrm{Zn}-\mathrm{Dy}$ and $\mathrm{Zn}-\mathrm{Dy}-$ $\mathrm{Zn}$ complexes with large anisotropy energy barriers: a combined theoretical and experimental magnetostructural study. Inorg. Chem. 55, 4428-4440. doi: 10.1021/acs.inorgchem.6b00228

Cucinotta, G., Perfetti, M., Luzon, J., Etienne, M., Car, P.-E., Caneschi, A., et al. (2012). Magnetic anisotropy in a dysprosium/DOTA single-molecule magnet: beyond simple magneto-structural correlations. Angew. Chem. Int. Ed. 51, 1606-1610. doi: 10.1002/anie.201107453

Dechambenoit, P., and Long, J. R. (2011). Microporous magnets. Chem. Soc. Rev. 40, 3249-3265. doi: 10.1039/c0cs00167h

Ding, Y.-S., Chilton, N. F., Winpenny, R. E. P., and Zheng, Y.-Z. (2016). On Approaching the Limit of molecular magnetic anisotropy: a near-perfect pentagonal bipyramidal dysprosium(III) single-molecule magnet. Angew. Chem. Int. Ed. 55, 16071-16074. doi: 10.1002/anie.201609685

Eerenstein, W., Mathur, N. D., and Scott, J. F. (2006). Multiferroic and magnetoelectric materials. Nature 442, 759-765. doi: 10.1038/nature05023

Ehama, K., Ohmichi, Y., Sakamoto, S., Fujinami, T., Matsumoto, N., Mochida, N., et al. (2013). Synthesis, structure, luminescent, and magnetic properties of carbonato-bridged ZnII2LnIII2 Complexes 


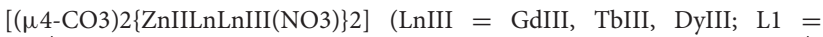
$\mathrm{N}, \mathrm{N}^{\prime}$-Bis(3-methoxy-2-oxybenzylidene)-1,3-propanediaminato, $\mathrm{L} 2=\mathrm{N}, \mathrm{N}^{\prime}$ Bis(3-ethoxy-2-oxybenzylidene)-1,3-propanediaminato). Inorg. Chem. 52, 12828-12841. doi: 10.1021/ic4022273

Escalera-Moreno, L., Baldoví, J. J., Gaita-Ariño, A., and Coronado, E. (2018). Spin states, vibrations and spin relaxation in molecular nanomagnets and spin qubits: a critical perspective. Chem. Sci. 9, 3265-3275. doi: 10.1039/C7SC05464E

Fiebig, M., Lottermoser, T., Meier, D., and Trassin, M. (2016). The evolution of multiferroics. Nat. Rev. Mater 1:16046. doi: 10.1038/natrevmats.2016.46

Goodwin, C. A. P., Ortu, F., Reta, D., Chilton, N. F., and Mills, D. P. (2017). Molecular magnetic hysteresis at 60 kelvin in dysprosocenium. Nature 548, 439-442. doi: 10.1038/nature23447

Gregson, M., Chilton, N. F., Ariciu, A.-M., Tuna, F., Crowe, I. F., Lewis, W., et al. (2016). A monometallic lanthanide bis(methanediide) single molecule magnet with a large energy barrier and complex spin relaxation behaviour. Chem. Sci. 7, 155-165. doi: 10.1039/C5SC03111G

Grünberg, P., Hüfner, S., Orlich, E., and Schmitt, J. (1969). Crystal field in dysprosium garnets. Phys. Rev. 184, 285-293.

Guo, F.-S., Day, B. M., Chen, Y.-C., Tong, M.-L., Mansikkamäki, A., and Layfield, R. A. (2017). A dysprosium metallocene single-molecule magnet functioning at the axial limit. Angew. Chem. Int. Ed. 56, 11445-11449. doi: 10.1002/anie. 201705426

Guo, F.-S., Day, B. M., Chen, Y.-C., Tong, M.-L., Mansikkamäki, A., and Layfield, R. A. (2018). Magnetic hysteresis up to 80 kelvin in a dysprosium metallocene single-molecule magnet. Science 362, 1400-1403. doi: 10.1126/science. aav0652

Gupta, S. K., Rajeshkumar, T., Rajaraman, G., and Murugavel, R. (2016). An air-stable Dy(iii) single-ion magnet with high anisotropy barrier and blocking temperature. Chem. Sci. 7, 5181-5191. doi: 10.1039/C6SC 00279J

Hang, T., Zhang, W., Ye, H.-Y., and Xiong, R.-G. (2011). Metal-organic complex ferroelectrics. Chem. Soc. Rev. 40, 3577-3598. doi: 10.1039/C0CS $00226 \mathrm{G}$

Horcajada, P., Gref, R., Baati, T., Allan, P. K., Maurin, G., Couvreur, P., et al. (2012). Metal-organic frameworks in biomedicine. Chem. Rev. 112, 1232-1268. doi: $10.1021 / \mathrm{cr} 200256 \mathrm{v}$

Ishikawa, N., Sugita, M., Ishikawa, T., Koshihara, S.-Y., and Kaizu, Y. (2003). Lanthanide double-decker complexes functioning as magnets at the singlemolecular level. J. Am. Chem. Soc. 125, 8694-8695. doi: 10.1021/ja029629n

Jia, J.-H., Li, Q.-W., Chen, Y.-C., Liu, J.-L., and Tong, M.-L. (2019). Luminescent single-molecule magnets based on lanthanides: design strategies, recent advances and magneto-luminescent studies. Coord. Chem. Rev. 378, 365-381. doi: 10.1016/j.ccr.2017.11.012

Leuenberger, M. N., and Loss, D. (2001). Quantum computing in molecular magnets. Nature 410, 789-793. doi: 10.1038/35071024

Li, B., Wen, H.-M., Cui, Y., Zhou, W., Qian, G., and Chen, B. (2016). Emerging multifunctional metal-organic framework materials. Adv. Mater. 28, 8819-8860. doi: 10.1002/adma.201601133

Liddle, S. T., and van Slageren, J. (2015). Improving f-element single molecule magnets. Chem. Soc. Rev. 44, 6655-6669. doi: 10.1039/C5CS00222B

Liu, J., Chen, Y.-C., Liu, J.-L., Vieru, V., Ungur, L., Jia, J.-H., et al. (2016). A stable pentagonal bipyramidal dy(iii) single-ion magnet with a record magnetization reversal barrier over 1000 K. J. Am. Chem. Soc. 138, 5441-5450. doi: $10.1021 /$ jacs.6b02638

Long, J., Basalov, I. V., Forosenko, N. V., Lyssenko, K. A., Mamontova, E., Cherkasov, A. V., et al. (2018a). Dysprosium single-molecule magnets with bulky schiff-base ligands: modification of the slow relaxation of the magnetization by substituent change. Chem. Eur. J. 25, 474-478. doi: 10.1002/chem.201804429

Long, J., Guari, Y., Ferreira, R. A. S., Carlos, L. D., and Larionova, J. (2018b). Recent advances in luminescent lanthanide based single-molecule magnets. Coord. Chem. Rev. 363, 57-70. doi: 10.1016/j.ccr.2018.02.019

Long, J., Guari, Y., Guerin, C., and Larionova, J. (2016a). Prussian blue type nanoparticles for biomedical applications. Dalton Trans. 45, 17581-17587. doi: 10.1039/C6DT01299J

Long, J., Mamontova, E., Freitas, V., Luneau, D., Vieru, V., Chibotaru, L. F., et al. (2016b). Study of the influence of magnetic dilution over relaxation processes in a $\mathrm{Zn} / \mathrm{Dy}$ single-ion magnet by correlation between luminescence and magnetism. RSC Adv. 6, 108810-108818. doi: 10.1039/C6RA $24115 \mathrm{H}$

Long, J., Rouquette, J., Thibaud, J.-M., Ferreira, R. A. S., Carlos, L. D., Donnadieu, B., et al. (2015). A high-temperature molecular ferroelectric $\mathrm{Zn} / \mathrm{Dy}$ complex exhibiting single-ion-magnet behavior and lanthanide luminescence. Angew. Chem. Int. Ed. 54, 2236-2240. doi: 10.1002/anie.201410523

Long, J., Vallat, R., Ferreira, R. A. S., Carlos, L. D., Almeida Paz, F. A., Guari, Y., et al. (2012). A bifunctional luminescent single-ion magnet: towards correlation between luminescence studies and magnetic slow relaxation processes. Chem. Commun. 48, 9974-9976. doi: 10.1039/c2cc35321k

Mandal, P., Pitcher, M. J., Alaria, J., Niu, H., Borisov, P., Stamenov, P., et al. (2015). Designing switchable polarization and magnetization at room temperature in an oxide. Nature 525, 363-366. doi: 10.1038/nature14881

Meng, Y.-S., Xu, L., Xiong, J., Yuan, Q., Liu, T., Wang, B.-W., et al. (2018). Low-coordinate single-ion magnets by intercalation of lanthanides into a phenol matrix. Angew. Chem. Int. Ed. 57, 4673-4676. doi: 10.1002/anie.201 801223

Oyarzabal, I., Ruiz, J., Ruiz, E., Aravena, D., Seco, J. M., and Colacio, E. (2015). Increasing the effective energy barrier promoted by the change of a counteranion in a Zn-Dy-Zn SMM: slow relaxation via the second excited state. Chem. Commun. 51, 12353-12356. doi: 10.1039/C5CC04495B

Pointillart, F., le Guennic, B., Cador, O., Maury, O., and Ouahab, L. (2015). Lanthanide ion and tetrathiafulvalene-based ligand as a "Magic" couple toward luminescence, single molecule magnets, and magnetostructural correlations. ACC. Chem. Res. 48, 2834-2842. doi: 10.1021/acs.accounts.5b00296

Ren, M., Bao, S.-S., Ferreira, R. A. S., Zheng, L.-M., and Carlos, L. D. (2014). A layered erbium phosphonate in pseudo-D5h symmetry exhibiting field-tunable magnetic relaxation and optical correlation. Chem. Commun. 50, 7621-7624. doi: 10.1039/C4CC02085E

Rinehart, J. D., and Long, J. R. (2011). Exploiting single-ion anisotropy in the design of f-element single-molecule magnets. Chem. Sci. 2, 2078-2085. doi: $10.1039 / \mathrm{c} 1 \mathrm{sc} 00513 \mathrm{~h}$

Shintoyo, S., Murakami, K., Fujinami, T., Matsumoto, N., Mochida, N., Ishida, T., et al. (2014). Crystal field splitting of the ground state of terbium(III) and dysprosium(III) complexes with a triimidazolyl tripod ligand and an acetate determined by magnetic analysis and luminescence. Inorg. Chem. 53, 10359-10369. doi: 10.1021/ic501453h

Sun, W.-B., Yan, P.-F., Jiang, S.-D., Wang, B.-W., Zhang, Y.-Q., Li, H.-F., et al. (2016). High symmetry or low symmetry, that is the question - high performance Dy(iii) single-ion magnets by electrostatic potential design. Chem. Sci. 7, 684-691. doi: 10.1039/C5SC02986D

Tang, J., and Zhang, P. (eds.). (2015). "Lanthanide single-ion molecular magnets," in Lanthanide Single Molecule Magnets (Berlin: Springer Berlin Heidelberg), 41-90. doi: 10.1007/978-3-662-46999-6

Train, C., Gruselle, M., and Verdaguer, M. (2011). The fruitful introduction of chirality and control of absolute configurations in molecular magnets. Chem. Soc. Rev. 40, 3297-3312. doi: 10.1039/c1cs15012j

Ungur, L., and Chibotaru, L. F. (2016). Strategies toward high-temperature lanthanide-based single-molecule magnets. Inorg. Chem. 55, 10043-10056. doi: 10.1021/acs.inorgchem.6b01353

Upadhyay, A., Singh, S. K., Das, C., Mondol, R., Langley, S. K., Murray, K. S., et al. (2014). Enhancing the effective energy barrier of a Dy(iii) SMM using a bridged diamagnetic $\mathrm{Zn}$ (ii) ion. Chem. Commun. 50, 8838-8841. doi: $10.1039 / \mathrm{C} 4 \mathrm{CC} 02094 \mathrm{D}$

Wang, H., Zhang, D., Ni, Z.-H., Li, X., Tian, L., and Jiang, J. (2009). Synthesis, crystal structures, and luminescent properties of phenoxo-bridged heterometallic trinuclear propeller- and sandwich-like schiff-base complexes. Inorg. Chem. 48, 5946-5956. doi: 10.1021/ic9002862

Wong, W.-K., Liang, H., Wong, W.-Y., Cai, Z., Li, K.-F., and Cheah, K.W. (2002). Synthesis and near-infrared luminescence of 3d-4f bi-metallic Schiff base complexes. New. J. Chem. 26, 275-278. doi: 10.1039/B10 $4175 B$

Wong, W.-K., Yang, X., Jones, R. A., Rivers, J. H., Lynch, V., Lo, W.-K., et al. (2006). Multinuclear luminescent schiff-base $\mathrm{Zn}-\mathrm{Nd}$ sandwich complexes. Inorg. Chem. 45, 4340-4345. doi: 10.1021/ic051866e

Woodruff, D. N., Winpenny, R. E. P., and Layfield, R. A. (2013). Lanthanide single-molecule magnets. Chem. Rev. 113, 5110-5148. doi: 10.1021/cr400018q 
Yamashita, K., Miyazaki, R., Kataoka, Y., Nakanishi, T., Hasegawa, Y., Nakano, M., et al. (2013). A luminescent single-molecule magnet: observation of magnetic anisotropy using emission as a probe. Dalton Trans. 42, 1987-1990. doi: $10.1039 / \mathrm{C} 2 \mathrm{DT} 32785 \mathrm{~F}$

Yang, X., and Jones, R. A. (2005). Anion dependent self-assembly of "TetraDecker" and "Triple-Decker" luminescent $\mathrm{Tb}(\mathrm{III})$ salen complexes. J. Am. Chem. Soc. 127, 7686-7687. doi: 10.1021/ja051292c

Yang, X., Jones, R. A., and Huang, S. (2014). Luminescent $4 \mathrm{f}$ and d-4f polynuclear complexes and coordination polymers with flexible salen-type ligands. Coord. Chem. Rev. 273-274. doi: 10.1016/j.ccr.2013.11.012
Conflict of Interest Statement: The author declares that the research was conducted in the absence of any commercial or financial relationships that could be construed as a potential conflict of interest.

Copyright (๑) 2019 Long. This is an open-access article distributed under the terms of the Creative Commons Attribution License (CC BY). The use, distribution or reproduction in other forums is permitted, provided the original author(s) and the copyright owner(s) are credited and that the original publication in this journal is cited, in accordance with accepted academic practice. No use, distribution or reproduction is permitted which does not comply with these terms. 